\title{
Autonomous assembly of synthetic oligonucleotides built from an expanded DNA alphabet. Total synthesis of a gene encoding kanamycin resistance
}

\author{
Kristen K. Merritt ${ }^{1,2}$, Kevin M. Bradley ${ }^{1,2}$, Daniel Hutter ${ }^{1,2,3}$, Mariko F. Matsuura ${ }^{1,2,4}$, \\ Diane J. Rowold ${ }^{1,2}$ and Steven A. Benner ${ }^{* 1,2,3}$
}

\author{
Full Research Paper \\ Address: \\ ${ }^{1}$ Foundation for Applied Molecular Evolution, P.O. Box 13174, \\ Gainesville, FL, 32604, USA, ${ }^{2}$ The Westheimer Institute for Science \\ and Technology, 720 S. W. 2nd Avenue, Suites 201-208, Gainesville, \\ FL, 32601, USA, ${ }^{3}$ Firebird Biomolecular Sciences LLC, 13709 \\ Progress Blvd. Box 17, Alachua, FL 32615, USA and ${ }^{4}$ Department of \\ Chemistry, University of Florida, Gainesville, FL, 32611, USA \\ Email: \\ Steven A. Benner* - sbenner@firebirdbio.com \\ * Corresponding author \\ Keywords: \\ automated gene synthesis; artificially expanded genetic information \\ systems; solid-phase DNA synthesis; synthetic biology
}

Beilstein J. Org. Chem. 2014, 10, 2348-2360. doi:10.3762/bjoc. 10.245

Received: 02 February 2014

Accepted: 18 September 2014

Published: 09 October 2014

Editor-in-Chief: P. H. Seeberger

(c) 2014 Merritt et al; licensee Beilstein-Institut. License and terms: see end of document.

\begin{abstract}
Background: Many synthetic biologists seek to increase the degree of autonomy in the assembly of long DNA (L-DNA) constructs from short synthetic DNA fragments, which are today quite inexpensive because of automated solid-phase synthesis. However, the low information density of DNA built from just four nucleotide "letters", the presence of strong (G:C) and weak (A:T) nucleobase pairs, the non-canonical folded structures that compete with Watson-Crick pairing, and other features intrinsic to natural DNA, generally prevent the autonomous assembly of short single-stranded oligonucleotides greater than a dozen or so.
\end{abstract}

Results: We describe a new strategy to autonomously assemble L-DNA constructs from fragments of synthetic single-stranded DNA. This strategy uses an artificially expanded genetic information system (AEGIS) that adds nucleotides to the four (G, A, C, and $\mathrm{T}$ ) found in standard DNA by shuffling hydrogen-bonding units on the nucleobases, all while retaining the overall Watson-Crick base-pairing geometry. The added information density allows larger numbers of synthetic fragments to self-assemble without off-target hybridization, hairpin formation, and non-canonical folding interactions. The AEGIS pairs are then converted into standard pairs to produce a fully natural L-DNA product. Here, we report the autonomous assembly of a gene encoding kanamycin resistance using this strategy. Synthetic fragments were built from a six-letter alphabet having two AEGIS components, 5-methyl-2'-deoxyisocytidine and 2'-deoxyisoguanosine (respectively $\mathbf{S}$ and $\mathbf{B}$ ), at their overlapping ends. Gaps in the overlapped assembly were then filled in using DNA polymerases, and the nicks were sealed by ligase. The $\mathbf{S}: \mathbf{B}$ pairs in the ligated construct 
were then converted to T:A pairs during PCR amplification. When cloned into a plasmid, the product was shown to make Escherichia coli resistant to kanamycin. A parallel study that attempted to assemble similarly sized genes with optimally designed standard nucleotides lacking AEGIS components gave successful assemblies of up to 16 fragments, but generally failed when larger autonomous assemblies were attempted.

Conclusion: AEGIS nucleotides, by increasing the information density of DNA, allow larger numbers of DNA fragments to autonomously self-assemble into large DNA constructs. This technology can therefore increase the size of DNA constructs that might be used in synthetic biology.

\section{Introduction}

It has been nearly 50 years since the first solid-phase synthesis of DNA by Letsinger and Mahadevan [1,2]. This work laid the platform for new strategies in oligonucleotide synthesis, culminating in the development of phosphoramidite-based synthesis of DNA in the 1980s [3]. The later full automation of these processes has caused many non-chemists to take, almost for granted, the low cost of synthetic DNA fragments. For example, when announcing the Defense Advanced Research Projects Agency's (DARPA) 2011 initiative in "Living Foundries," the program manager is quoted as saying that "DNA should cost next to nothing" [4]. Indeed, the DARPA Foundries 1000 molecules program hopes for the assembly of large DNA (L-DNA) "chassis" via fully automated processes.

Historically, essentially all syntheses of L-DNA constructs, including synthesis of complete genes, have followed a convergent assembly strategy. This was used for the first time for the total synthesis of genes encoding transfer RNA [5], but convergent synthesis is generally routine for the synthesis of natural products [6]. In the convergent synthesis of L-DNA, subsets of designed single-stranded fragments are first assembled to give parts of a longer target that may (or may not) have their structures determined. The fragments are subsequently combined to create still longer parts, often by joining sticky ends generated by partial digestion of the ends of the duplex strands [7]. The cycle is then repeated until the full-length L-DNA product is achieved.

With the advent of PCR, this strategy was adapted to the total synthesis of a gene encoding human leukocyte interferon [8] and a gene encoding ribonuclease $\mathrm{S}$ protein [9]. The second gene synthesis was the first to exploit the phosphoramidite synthesis approach developed in the Caruther laboratory [3], and also the first to illustrate the ability of total synthesis to generate DNA products that are productively different from what might be found in nature. Total synthesis was used to introduce restriction sites to facilitate subsequent manipulation, add 'watermarks' to track the gene's provenance, and choose codons to improve the expression of the gene [10].
This combination of automated synthesis of DNA fragments followed by their manual assembly, with PCR used to recover manually assembled partial constructs, began a three decade long effort that has continued to push the limits of synthesis and convergent assembly. One recent example is the synthesis using convergence of a DNA molecule that exactly reproduced (except for watermarks) the natural genome of Mycobacterium genitalium [11]. Although the power of synthesis was not exploited to place innovative features into the synthetic $M$. genitalium genome, the use of cells to assist in the assembly of synthetic fragments represented a technological advance.

In all of these efforts, while the synthesis of fragments was automated, the subsequent assembly of parts from them, and the assembly of larger parts from smaller parts, was not. While costs estimates vary, the paper reporting the convergent assembly of the Mycobacterium genome had 17 coauthors and represented millions of dollars of expense [11]. More recently, a functional 272,871 base-pair designer chromosome was assembled based on a yeast chromosome [12]. The sequence of chromosome III of Saccharomyces cerevisiae was edited with insertions and deletions to give a smaller "synIII" product. This expensive project engaged a team of more than 70 coauthors.

These heroic accomplishments have driven the vision, now called "synthetic biology" [13-17], of converting automatically synthesized short DNA molecules (50-100 nucleotides in length) into much longer DNA constructs by autonomous selfassembly, without the cost of manual convergent assembly. In addition to the DARPA Foundries "1,000 molecules" strategy mentioned above, the United States Army Research Office in 2011 issued a small business grant solicitation seeking software to allow 30,000 base pairs of single-stranded DNA to selfassemble to form nanostructures. In 2012, DARPA issued a small business grant solicitation seeking technology to assemble single-stranded synthetic fragments to give 20,000 base-pair DNA constructs.

If DNA were in fact the idealized molecule taught in introductory biochemistry classes, the specificity of Watson-Crick 
nucleobase pairing might indeed allow autonomous assembly of large numbers of synthetic single strands to give such large targets, with only a modest amount of design to ensure minimal off-target annealing. With ideal DNA, plasmid-sized constructs (L-DNA, 1,000-10,000 base pairs) and possibly even ultra-long DNA constructs (UL-DNA > 10,000 base pairs) might assemble simply by combining the requisite single-stranded oligonucleotides followed by annealing, primer extension to fill in any gaps, and ligation.

Unfortunately, DNA is not this ideal. With just four nucleotides, the information density of standard DNA is too low to allow (without explicit design) even a dozen or so single strands to reliably self-assemble upon simple mixing. Even with explicit design, the number of fragments that can be self-assembled appears to be not much larger. This is because of properties intrinsic in the structure of the nucleobases themselves. First, even if rule-based Watson-Crick pairing were the only possible interaction, the presence of "strong" and "weak" G:C and A:T pairs makes design challenging. Also complicating selfassembly of single-stranded DNA fragments are folded single-strand structures (such as hairpins) that compete with desired inter-strand hybridization. A rich repertoire of nonWatson-Crick interactions (e.g., wobble, major groove binding) can also compete with Watson-Crickery (Figure 1).

The work reported here began with the observation that several of the structural features of DNA that intrinsically limit autonomous assembly of standard DNA fragments might be overcome by adding non-standard nucleotides to the repertoire introduced into DNA strands by automated synthesis. These additional non-standard nucleotides come from components of an artificially expanded genetic information system (AEGIS) $[18,19]$.

AEGIS adds nucleotides to the DNA alphabet by rearranging the hydrogen-bonding units displayed by the nucleobases, allowing them to pair orthogonally within the geometry of the Watson-Crick pair (Figure 2). With higher information density in AEGIS, more fragments should self-assemble with fewer off-target hybridization, fewer hairpins, and fewer close mismatches that slow down annealing.

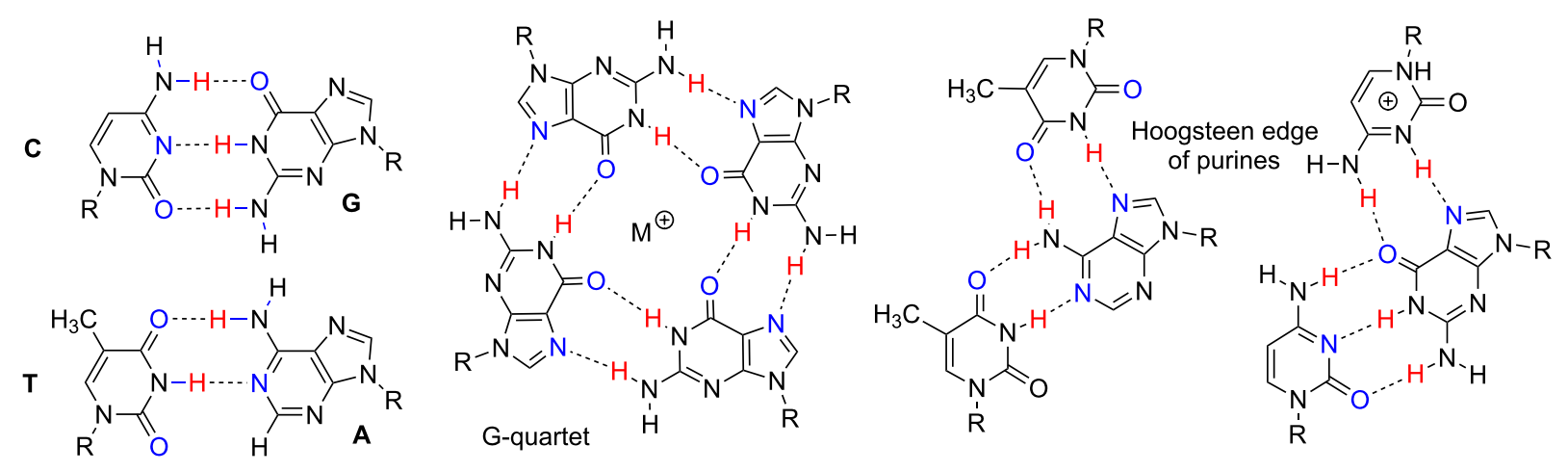

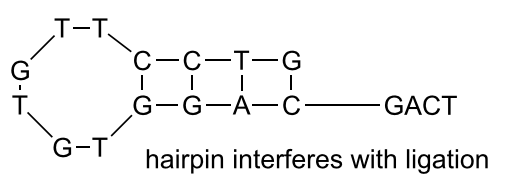

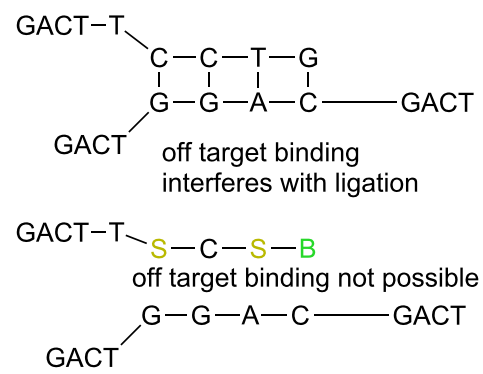

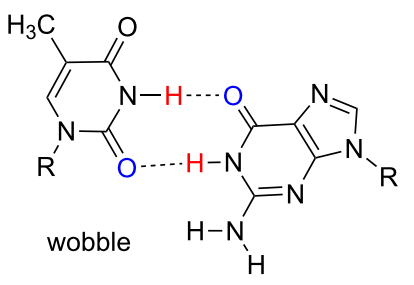

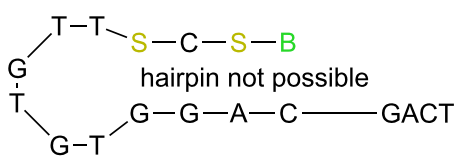

GACT

Figure 1: Structures explaining why large DNA (L-DNA) constructs cannot easily autonomously self-assemble from many synthetic single-stranded DNA oligonucleotides. Clockwise from top left, the strong $C: G$ and weak $T: A$ pairs complicate design. G-quartets can arise from $G$-rich sequences. Major groove interactions involving hydrogen bonding to the "Hoogsteen edge" of purines can compete with Watson-Crickery. Wobble pairs allow formal mismatches to nonetheless contribute to duplex stability, depending on context. The low information density of four-nucleotide DNA allows easy off-target hybridization and unimolecular formation of hairpins. Unimolecular processes (like hairpin formation) compete with the desired intermolecular hybridization especially effectively at low concentrations of oligonucleotide. Adding components of an artificially expanded genetic information system (AEGIS, here $\mathbf{S}$ and $\mathbf{B}$ ) prevent a class of these obstructive structures. 


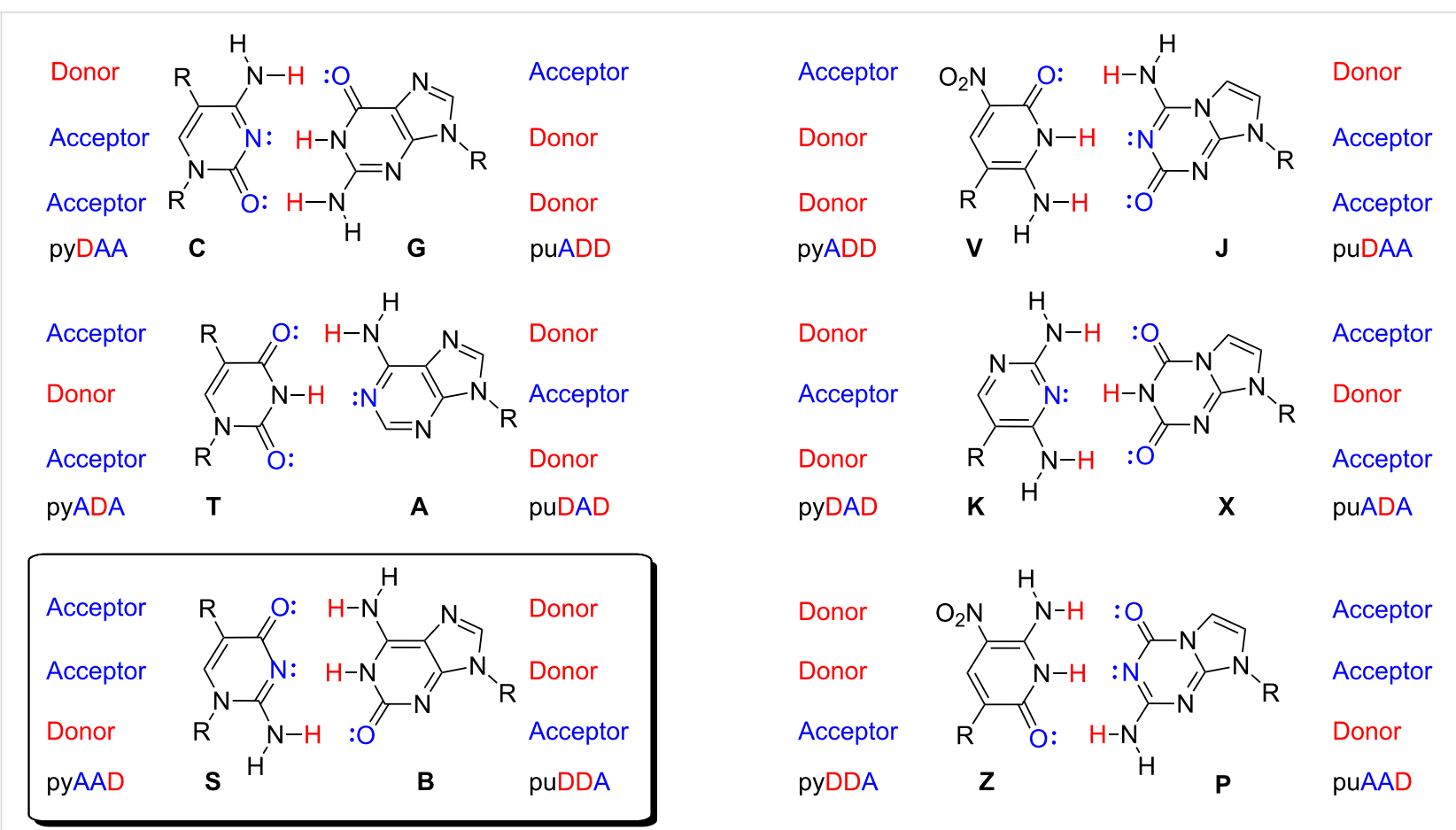

Figure 2: Watson-Crick pairing rules follow two rules of complementarity: (a) size complementarity (large purines pair with small pyrimidines) and (b) hydrogen-bonding complementarity (hydrogen-bond acceptors, A, pair with hydrogen-bond donors, D). Rearranging donor and acceptor groups on the nucleobases, while not changing the geometry of the Watson-Crick pair, creates an artificially expanded genetic information system (AEGIS). AEGIS components add information density to DNA strands built from them. The S:B pair used in this work is highlighted in the box.

Of course, the resulting assembly has unnatural AEGIS components, which may not be desired in the final L-DNA construct. The recently developed conversion technology [20] solves this problem for certain AEGIS pairs. Conversion occurs when polymerases are forced to mismatch a standard nucleotide opposite an AEGIS nucleotide by (a) not being provided the complementary AEGIS triphosphate and (b) exploiting a chemical feature of the AEGIS nucleotide that directs a specific mismatch.

For example, when the AEGIS nucleotide 2'-deoxy-5methylisocytidine (trivially designated $\mathbf{S}$ ) and its AEGIS complement 2'-deoxyisoguanosine (trivially designated B) (Figure 2) are used to assemble a L-DNA construct, the S:B pair forms orthogonally to the T:A and C:G pairs $[18,19]$. However, if $\mathrm{dSTP}$ is missing during primer extension, $\mathbf{B}$ in a template must be mismatched during primer extension. Here, conversion exploits the ability of a minor tautomer of $\mathbf{B}$ to mismatch with standard T (Figure 3), After this initial mismatch, the misincorporated $\mathrm{T}$ directs the incorporation of $\mathrm{dATP}$, resulting in a net conversion of the $\mathbf{S}: \mathbf{B}$ pairs in the preliminary construct to $\mathrm{T}: \mathrm{A}$ pairs in the final construct (Figure 3) [20]. This conversion can be performed in vitro, but also in E. coli in a strain (SEGUE) being developed to replicate plasmids containing expanded genetic systems.
Here, we illustrate this strategy by performing a total synthesis of a gene encoding an aminoglycoside 3'-phosphotransferase that confers kanamycin resistance by autonomous selfassembly, where $\mathbf{S}: \mathbf{B}$ pairs used to create the clean fragment assembly are later converted via this process to T:A pairs. Figure 4 shows the details of the assembly.

\section{Results}

\section{The OligArch software package and the construct design}

To support this project, a software package (OligArch) [21] was created to design DNA molecules that exploit AEGIS nucleotides to facilitate the self-assembly of multiple singlestranded DNA. The OligArch package takes as input a target sequence for a desired long DNA (L-DNA) construct. It then fragments the target to deliver, as output, a set of singlestranded DNA molecules that include AEGIS components. OligArch designs these fragments so that, after they are annealed, the annealed fragments are extended by a DNA polymerase to fill in any gaps, the nicks in the resulting duplex are ligated (Figure 3 schematically, Figure 4 in detail), and the AEGIS pairs are replaced by standard pairs by conversion PCR, all to produce the desired L-DNA construct to emerge with only standard bases. To meet these performance specifications, OligArch must know the rules for conversion of AEGIS 


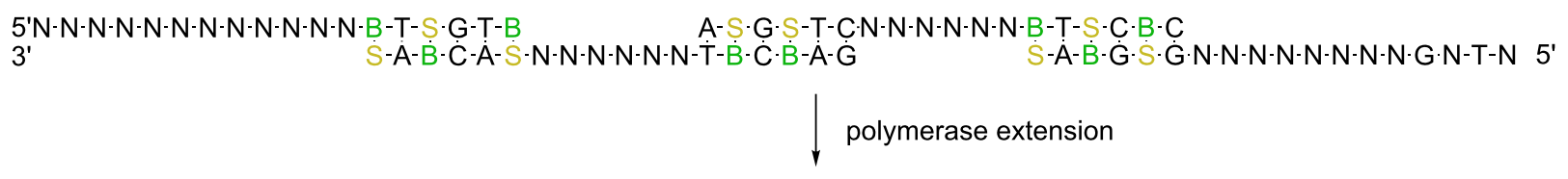

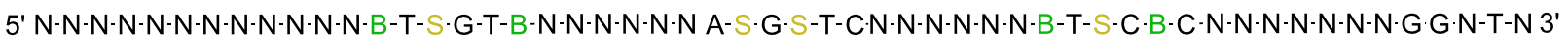

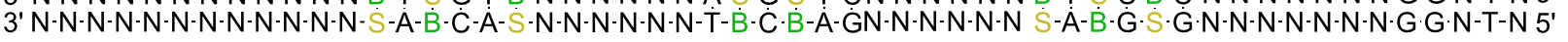

$$
\downarrow \text { ligation }
$$

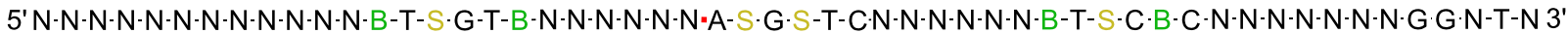
$3^{\prime} N \cdot N \cdot N \cdot N-N \cdot N \cdot N \cdot N \cdot N \cdot N \cdot N \cdot N-S \cdot A-B \cdot C \cdot A-S \cdot N \cdot N \cdot N \cdot N \cdot N \cdot N-T \cdot B \cdot C \cdot B-A \cdot G N \cdot N \cdot N \cdot N \cdot N \cdot N \cdot S-A-B \cdot G \cdot S \cdot G \cdot N \cdot N \cdot N \cdot N \cdot N \cdot N \cdot N \cdot G \cdot G \cdot N-T \cdot N 5 \cdot$

$$
\downarrow \text { PCR amplification with conversion }
$$

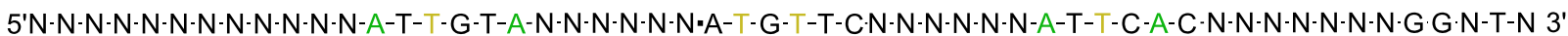

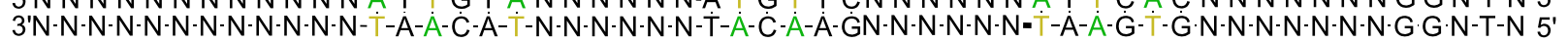

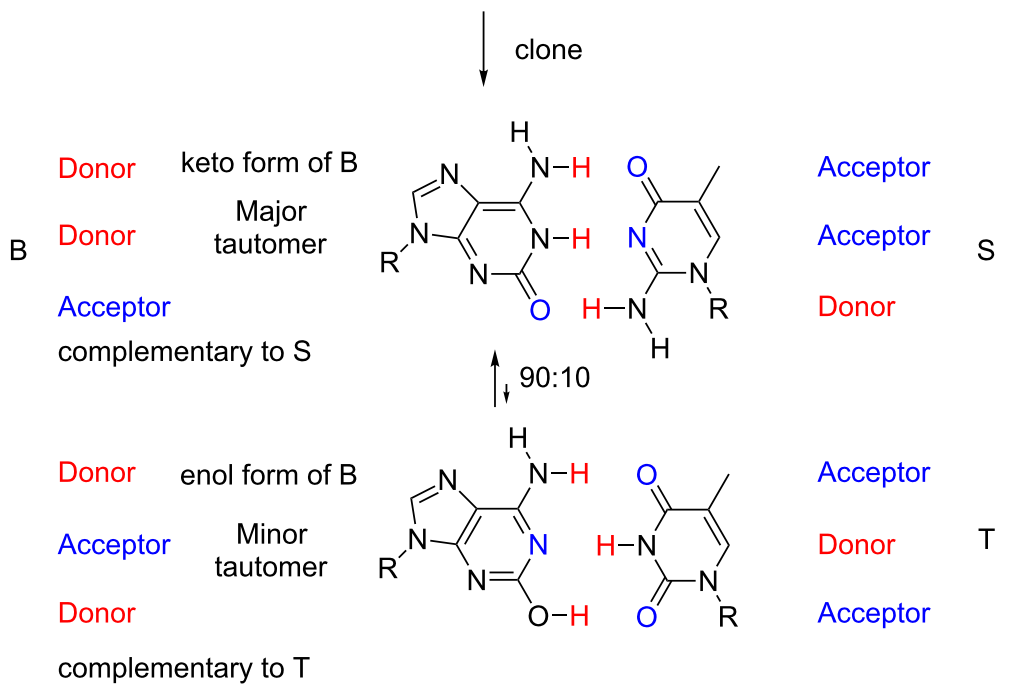

Figure 3: (top) Schematic showing the mix-anneal-extend-ligate-amplify process exploiting AEGIS pairing (to obtain clean assembly) followed by conversion to remove AEGIS pairs from the final construct. The process starts by mixing single-stranded DNA fragments designed to have their ends anneal as duplexes that include S:B pairs (note the colors). The higher information density enabled by the additional S:B pair lowers off-target hybridization, eliminates hairpins, and better guides the formation of the desired duplexes. After these duplexes are formed, the 3'-ends are extended using a polymerase that does not displace strands to give nicked DNA. Ligase then seals the nicks by forming the red bonds. Then, PCR amplification with conversion (shown here) or direct transformation into SEGUETM replaces the $\mathbf{S}: \mathbf{B}$ pairs in the construct by $\mathrm{T}: \mathrm{A}$ pairs. (bottom) $\mathbf{B}$ in its major tautomeric form pairs with $\mathbf{S}$; in its minor tautomeric form, B pairs with standard T. This allows the AEGIS $\mathbf{S}: \mathbf{B}$ pair to support the assembly of multiple single-stranded fragments using the mix-anneal-extend-ligate-amplify process, to be followed by conversion of the $\mathbf{S}: \mathbf{B}$ pairs to T:A pairs after two cycles of PCR. In the conversion template $\mathbf{B}$ is replaced by standard A via an intermediate $\mathbf{B}: \mathrm{T}$ mispairing, while template $\mathbf{S}$ is replaced by $\mathbf{T}$ via an intermediate $\mathbf{S}: \mathbf{B}$ pairing followed by a second intermediate $\mathbf{B}: \mathbf{T}$ mispairing.

nucleotides to standard nucleotides; these are provided from experimental data.

OligArch also allows the user to designate certain regions as protein-encoding sites; at these sites, redundancy in the genetic code can be exploited. Sites may also be designated where no flexibility is permitted. OligArch seeks to have desired duplexes formed with a melting temperature not lower than a user-specified threshold temperature. Once this temperature is specified, OligArch automatically designs the fragments so that the best off-target hybridizations have melting temperatures below a second "off-target" level.

This difference (or "spread") between the melting temperature of the "weakest wanted" and "strongest unwanted" pairings is a predictor of the success of autonomous assembly; the larger the spread, the more likely the self-assembly will succeed. With just the four standard nucleobases, the spread cannot remain large as the number of fragments increases. Accordingly, various practitioners recommend attempting self-assembly with 


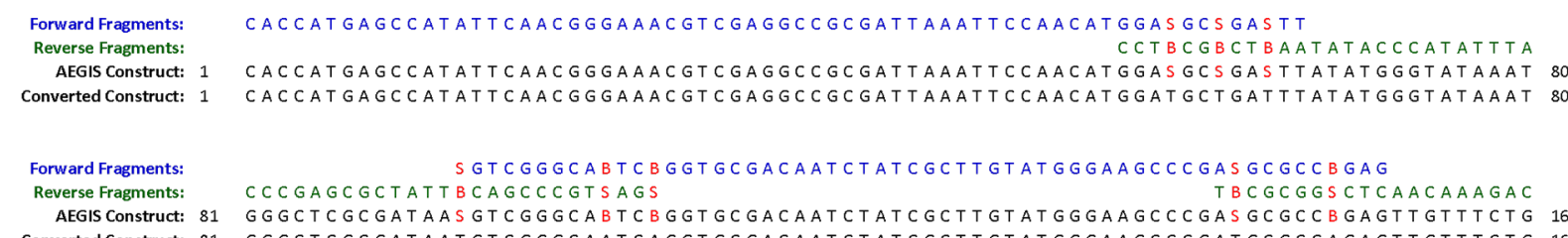

AEGIS Construct: 81 GGGCTCGCGATAASGTCGGGCABTCBGGTGCGACAATCTATCGCTTGTATGGGAAGCCGASGCGCCBGAGTGTTTCTG 160 Converted Construct: 81 GGGCTCGCGATAATGTCGGGCAATCAGGTGCGACAATCTATCGCTTGTATGGGAAGCCGATGCGCCAGAGTTGTTTCTG 160

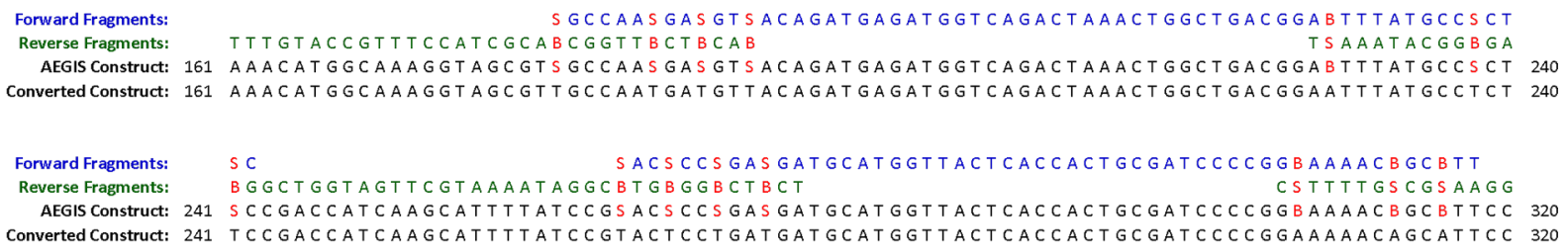

Figure 4: The exact sequences and overlaps of the fragments designed by OligArch to allow the autonomous assembly of a gene encoding an aminoglycoside 3'-phosphotransferase that confers upon E. coli resistance to kanamycin, aligned above the gene that arises via conversion PCR. The AEGIS nucleotides, $\mathbf{S}$ and $\mathbf{B}$ (red), were placed in the overlap regions to guide self-assembly.

no more than a dozen or so fragments [22], although circa three dozen have been assembled inside of yeast cells [23].

In this work, we chose $\mathbf{S}$ (2'-deoxy-5-methylisocytidine) and $\mathbf{B}$ (2'-deoxyisoguanosine) as the AEGIS nucleotides; adding these gives a six-letter GACTSB DNA alphabet. This was an alternative to the AEGIS nucleotides 2-amino-8-(1'- $\beta$-D-2'-deoxyribofuranosyl)imidazo[1,2- $a]$-1,3,5-triazin-4( $8 H)$-one (trivially named $\mathbf{P}$ ) and 6-amino-5-nitro-3-(1'- $\beta$-D-2'-deoxyribofura-
nosyl)-2(1H)-pyridone (trivially named $\mathbf{Z}$ ) (Figure 2), which give a GACTZP six-letter DNA alphabet. This choice reflected simpler conversion rules, to be discussed elsewhere.

\section{AEGIS-guided oligonucleotide assembly yields a kanamycin resistance gene}

The actual sequences designed by OligArch and used here are shown in Figure 4, with overlaps. These were prepared by automated DNA synthesis from six phosphoramidites (four stan- 
dard and two AEGIS). They were then mixed in equal amounts, heated and cooled. The 3'-fragments were then extended at $48{ }^{\circ} \mathrm{C}$ using Phusion DNA polymerase to give a nicked construct and the nicks were sealed with ligase.

The first indication that the GACTSB AEGIS self-assembly was successful was the electrophoretic detection of the fulllength non-amplified product (Figure 5). A gel resolving crude, non-amplified products obtained from the "one-pot" annealing, extension, and ligation process showed a major band at $\sim 863$ base pairs, the size of the expected product (Figure 5). This suggested that the 20 fragments with AEGIS overhangs selfassembled to give the target gene as one of the principal products. As discussed in our comparative study (see below), this is rarely seen in mixtures of fragments built from the standard GACT DNA alphabet, even with fewer fragments.

The product of the autonomous assembly was then ligated behind a $\beta$-galactosidase promoter in a plasmid containing a gene conferring resistance to ampicillin. This was used to transform E. coli cells, which were found to grow in medium containing kanamycin. Plating experiments quantitated these results (Figure 6). Plates with kanamycin but no IPTG (which induces expression of the synthetic gene) gave no colonies (Figure 6c). With IPTG, however, multiple colonies grew (Figure 6b) in the presence of kanamycin, with the expected smaller size than the colonies seen on plates lacking kanamycin altogether (Figure 6a).

These results demonstrated successful autonomous assembly of a gene encoding an active aminoglycoside 3'-phosphotransferase. They allowed us to estimate the success of the overall

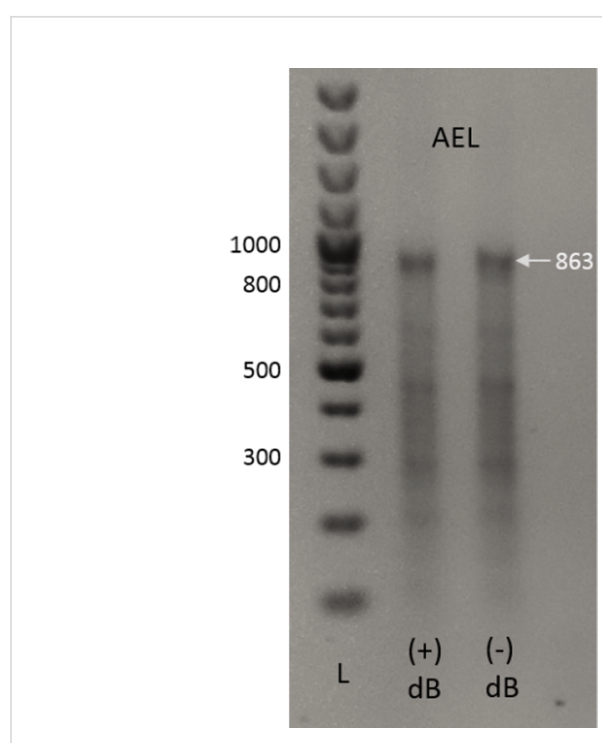

Figure 5: Agarose gel (1\%, stained with ethidium bromide) showing the products (before PCR) arising from the autonomous assembly of the AEGIS-containing oligonucleotide fragments (Figure 4) for a gene encoding kanamycin resistance (863 base pairs) under two incubation conditions (\#1, same method as in text with 2'-d-isoGTP (dBTP) included in the mixture; \#2 as in text). Left lane (L) is a ladder with markers indicated at $300,500,800$, and 1,000 nucleotides. Remarkably, a principal product runs at 863 nucleotides, the length of the complete construct arising from the autonomous assembly of 20 fragments. When similar autonomous assembly without AEGIS nucleotides is attempted, the desired full-length product is almost never seen as a principal product, and almost never recovered without PCR.

"start-to-finish" process, including insertion into the plasmid and other steps unrelated to the OligArch GACTSB AEGIS assembly. The primary culture contained approximately 23,000 kanamycin-resistant cells per $\mathrm{mL}$. The same culture contained
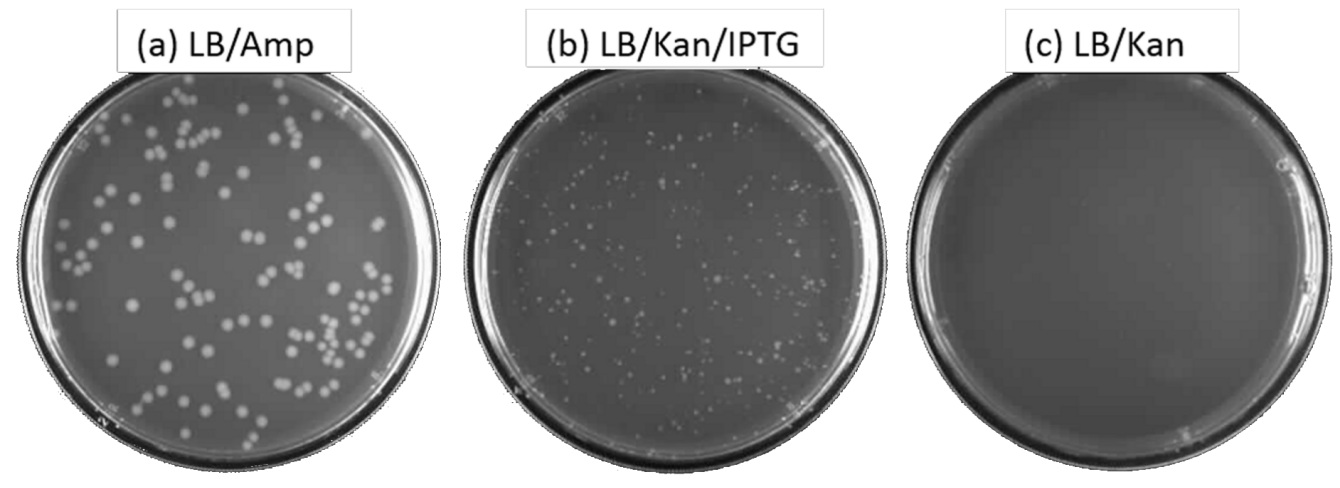

Figure 6: LB-agar plates spread, at various dilutions, with $25 \mu \mathrm{L}$ cells transformed by plasmids containing the self-assembled kanamycin-resistance gene placed behind a $\beta$-galactosidase promoter in the TOPO expression vector (Invitrogen), but without selection for resistance to kanamycin. (a) The cell culture was diluted in two steps by a factor of 267,000 . Then, aliquots $(25 \mu \mathrm{L})$ were plated on agar containing only ampicillin. Replica plates (only one is shown) gave 123 and 99 colonies (average is 111 colonies), for a calculated $29.0 \times 10^{6}$ ampicillin-resistant colonies/mL in the original cell culture. (b) The cell culture was diluted by a factor of 2 . Then, $25 \mu \mathrm{L}$ was plated on agar containing IPTG and kanamycin. Replica plates (only one is shown) gave 308 and 276 colonies (average is 292 colonies), for a calculated 23,000 kanamycin-resistant colonies/mL in the original cell culture. (c) With insert but without IPTG induction of the gene for kanamycin resistance, no growth is seen. 
approximately 29,000,000 total cells per mL. This suggests that the overall process generated successfully constructed, inserted, converted, and successfully expressed kanamycin-resistance genes in $\sim 0.1 \%$ of the cells that had acquired ampicillin resistance by transformation.

The final demonstration of the success of the GACTSB autonomous assembly came, of course, from the sequencing of the cloned product. Sequencing also allowed us to estimate the error associated with the process. Table S1 (Supporting Information File 1) collects a set of sequences of the constructs that were created by the procedure where dBTP was used in the conversion procedure (see Experimental). While 1-2\% errors were seen, none were found in the sites where $\mathbf{S}: \mathbf{B}$ pairs had been present. The errors were found most frequently at the ends of the reads, as expected for sequencing errors, rather than errors in the primary synthesis, polymerase extension, or conversion PCR.

\section{Standard oligonucleotide assembly attempts without AEGIS components fail}

While these results show that synthetic DNA fragments can self-assemble to give complete genes, they also raise the question: Are AEGIS components needed? Can we not assemble genes of the same size with only standard nucleotides, without any AEGIS components at all?

It appears that no systematic experiments have been performed to benchmark the limits to autonomous self-assembly of natural DNA. Therefore, we undertook a series of "push to fail" experiments where we attempted to prepare larger and larger assemblies from more and more fragments constructed from standard nucleotides, using three sets of fragments with up to 32 fragments in each set. Each set of fragments was designed by OligArch with only one goal in mind: to give autonomous assembly the best chance of success. To this end, OligArch used three different "seeds" to initiate the fragment design [21]. The fragments were designed to have nearly identical lengths (50-52 nts) and 15-17 nucleotide overlap with melting temperatures predicted to lie in a narrow range $\left(44-56{ }^{\circ} \mathrm{C}\right.$; calculated without magnesium). OligArch also designed the sequences to have no-off target hybrids having a melting temperature greater than $25{ }^{\circ} \mathrm{C}$, a full $20^{\circ} \mathrm{C}$ below that predicted for the desired annealing pairs.

Two of the three constructs (" $32 \mathrm{~B}$ " and "32C") contained the four standard nucleotides, G, A, T, and C throughout. In the third construct ("32A"), OligArch placed AEGIS nucleotides $\mathbf{S}$ and $\mathbf{B}$ (Figure 2) in the overlapping regions to facilitate selfassembly. Figures S1-S3 (Supporting Information File 1) show the designed single-stranded DNA fragments and their hybridizing segments. All three constructs were designed to have approximately $1.1 \mathrm{~kb}$ pairs when fully assembled (Supporting Information File 1, Tables S7-S9).

The results are consistent with the discussion above. With the $32 \mathrm{~A}$ construction (Supporting Information, Figure S1) that did exploit the AEGIS S:B pair (Figure 2) in the annealing overhang segments, a PCR product arising from end-to-end PCR (with conversion of the $\mathbf{S}: \mathbf{B}$ pairs to T:A pairs) with the expected length of 1,121 base pairs was immediately seen (Figure 7). The product was directly cloned and sequenced, to prove success of the AEGIS-assisted autonomous assembly.

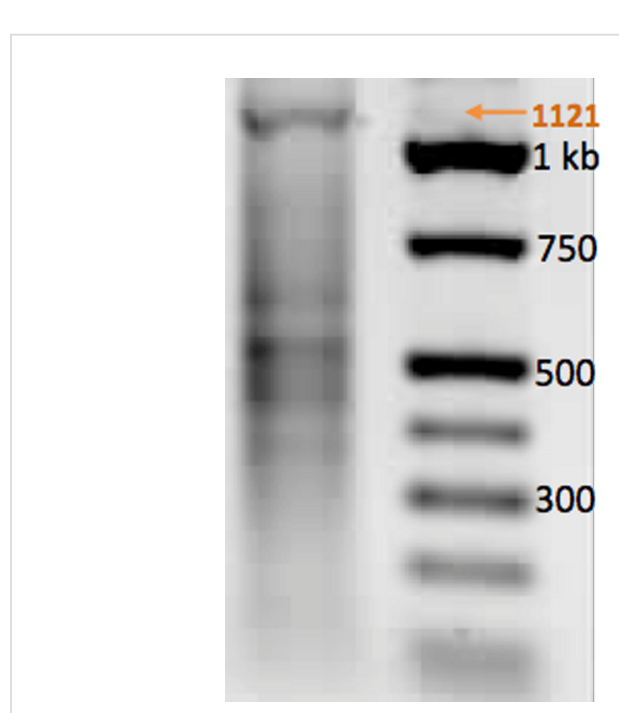

Figure 7: (left) Autonomous assembly of the 32A construct yielded the desired 1,121 base-pair amplicon in a single step involving mixing, annealing, extending, and ligation, followed by PCR amplification (30 cycles). Orange arrow shows the full length product obtained. (right) Ladder of synthetic fragments having lengths indicated (nucleotides).

With the 32B construct that lacked AEGIS nucleotides, no fulllength product was observed when all 32 fragments were mixed without amplification (data not shown). To rule out the possibility that the oligonucleotides were defective, smaller constructs were self-assembled. Figure 8 shows the results of stepwise assembly of subsets of the fragments, after the target ligation products were rescued from the mixture by PCR ( 30 cycles). As is evident by the intensity of the bands arising from the amplicons four, eight, and twelve fragments easily assembled without the need for any AEGIS nucleotides to guide that assembly. Sixteen fragments also assembled, but the yield of the assembled construct was approximately $30 \%$ lower. An assembly of 20 fragments could be only be recovered by PCR under different conditions (data not shown). Attempts to assemble 24 to 32 fragments failed to yield any detectable amplicon. 


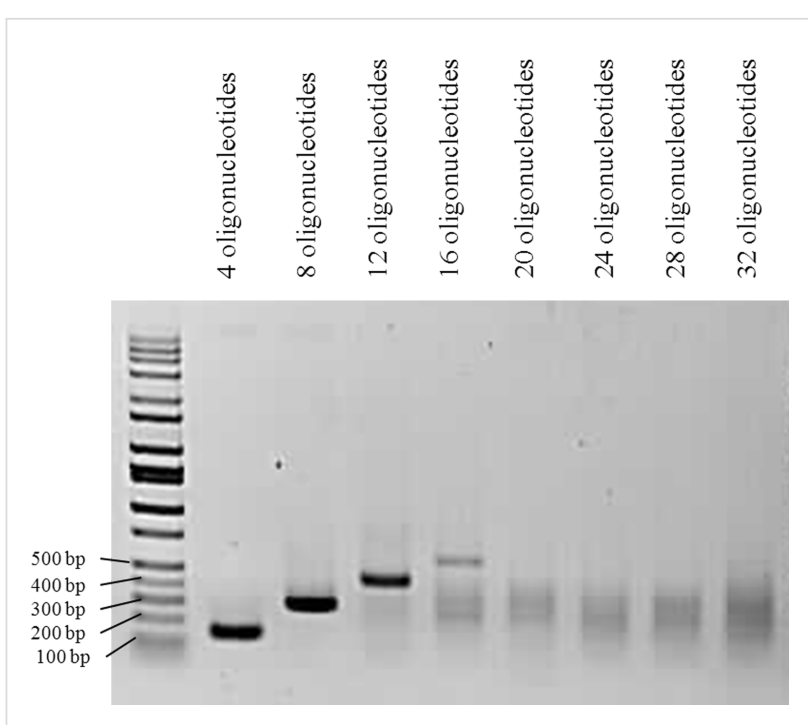

Figure 8: Fragments built only from standard nucleotides, without AEGIS, and designed by OligArch [21] to support the 32B assembly (Supporting Information File 1, Figure S2) resulted in successful selfassembly of up to 16 pieces, in decreasing amounts as the constructs became longer, where the 16 -fragment assembly was recovered by PCR. However, under these conditions PCR failed to recover longer assemblies built from $20,24,28$, or 32 fragments. This represents an experience common when attempting autonomous assembly of DNA single strands. Shown is a $1 \%$ agarose gel resolving those products, with ladder at left.

However, the two halves of the 1,135 base-pair construct, once separately assembled and amplified by PCR to create the half assemblies in large amounts, could then be manually joined in a standard convergent synthesis after blunt-end ligation. The desired 1,135 base-pair target construct was recovered by PCR (see Supporting Information File 1, Figure S4). This process was, of course, not automated; it represented the same stepwise convergent assembly of L-DNA that has been reported previously [3-5,9].

The 32C assembly attempt also failed initially. However, upon increasing the concentrations of oligonucleotide fragments from $62.5 \mathrm{nM}$ to $125 \mathrm{nM}$, followed by PCR amplification, a PCR product of the desired length could occasionally be recovered (Supporting Information File 1, Figure S5).

\section{Discussion}

Adding nucleotide "letters" to an expanded genetic "alphabet" increases the information density of the resulting DNA sequences. With four nucleotides, the number of overlapping 15 mers is ca. 1.1 billion $\left(\approx 4^{15}\right)$. While this number might appear to be large, it includes an enormous range of melting temperatures, because the G:C and A:T pairs contribute differently to the stability of a duplex. Adding two additional nucleotides increases the number of potential overlaps to 470 billion $\left(\approx 6^{15}\right)$, more than 400 fold higher.
The increased information density is expected a priori to improve the ability of single-stranded fragments to selfassemble without human guidance. Further, the $\mathbf{S}: \mathbf{B}$ pair with its three hydrogen bonds is "strong," like the G:C pair. This means that the OligArch design of fragments can focus on obtaining the best chance of delivering the longest possible desired sequence, rather than accommodating another weak nucleobase pair.

Further, $\mathbf{S}$ and $\mathbf{B}$ can be used strategically, as illustrated in the synthesis of the kanamycin gene. Here, $\mathbf{S}$ and $\mathbf{B}$ were placed at the ends of the single-stranded oligonucleotides, and not in the central portions of those oligonucleotides. This makes it essentially impossible for intramolecular hairpins to compete with the intermolecular annealing (Figure 1). Intramolecular folding is expected to be especially destructive as the number of fragments increases, if the concentration of each individual fragment decreases.

In "push to fail" experiments that compared the assembly of fragments that exploit AEGIS components with assemblies that do not, the improvement could be assessed. Thus, the 32B assembly of entirely standard oligonucleotides, designed with no constraint other than to optimize self-assembly, worked with up to 16 fragments (but not longer) (Figure 8). With higher concentrations of fragments $(125 \mathrm{nM})$, the $32 \mathrm{C}$ assembly, upon PCR rescue, sometimes yielded constructs more easily than the 32B assembly (Supporting Information File 1, Figure S5). While folds and other non-canonical secondary structures (Figure 1 and Supporting Information File 1, Figure S6) may have prevented the $32 \mathrm{~B}$ assembly from working, comparable folds can be found for any set of fully standard fragments.

Further, real sequences intended to advance a synthetic biology agenda are rarely ideal; their design is likely constrained in a way that, without the help of AEGIS nucleotides, will prevent OligArch from achieving the large differences in melting temperatures, and achieving total avoidance of hairpins and other secondary structures that obstruct autonomous assembly.

These results provide, therefore, a realistic view of what automated DNA synthesis can provide by way of products that can be carried forward in an automated fashion to make larger products. The use of AEGIS nucleotides enables a breakthrough of the "dozen fragment barrier" and facilitates the modular formation of L-DNA constructs $(>1 \mathrm{~kb})$. Other factors will now become limiting for the self-assembly of L-DNA constructs, including errors in the synthesis of the DNA fragments used for the assembly. These must be mitigated in any effort to look forward, especially in the hope of finding inexpensive automated strategies that assemble inexpensive 
oligonucleotides into long DNA constructs (L-DNA) and (possibly) ultralong DNA (UL-DNA) constructs, including whole chromosomes.

\section{Experimental \\ Materials and methods \\ Design}

The sequence for an aminoglycoside 3'-phosphotransferase that encodes resistance to kanamycin is commonly used in cloning vectors and was obtained from the National Center for Biotechnology Information database (NCBI). Using the OligArch software designed for this purpose [21], a gene that encodes the protein was constructed to be assembled from 20 singlestranded DNA fragments containing two, three, or four $\mathbf{S}$ (2'deoxy-5-methylisocytidine) and B (2'-deoxyisoguanosine) nucleotides at their 5' and 3' ends. To obtain consistent melting temperatures, OligArch designed oligonucleotides ranging in size from 41 to 68 nucleotides (nts) (Table 1). Overlaps were 11-15 nucleotides in length, and designed to have theoretical melting temperatures of 42 and $52{ }^{\circ} \mathrm{C}$ (calculated at $50 \mathrm{mM}$ $\mathrm{NaCl}$ without divalent magnesium; using the nearest neighbor formula from http://www.clinchem.org/content/45/12/ 2094.long; melting temperatures in $10 \mathrm{mM} \mathrm{MgCl} 2$ are expected to be $\sim 10^{\circ} \mathrm{C}$ higher) The $\mathbf{S}: \mathbf{B}$ pair was assumed to contribute to duplex stability the same as the C:G pair. Enthalpy and entropy values for nucleotide pairs in context were taken from [24].
Additionally, the L-DNA product was designed to have a CACC tetranucleotide immediately upstream from the start codon; this assisted incorporation into a TOPO expression vector. The synthetic segment was also designed to have a Bam HI region downstream of the stop codon, also to assist in downstream processing. Thus, the complete target L-DNA sequence had 863 base pairs.

\section{Synthesis}

The oligonucleotides containing $\mathbf{S}$ and $\mathbf{B}$ shown in Table 1 were obtained from IDT, where they were prepared by automated solid phase phosphoramidite-based synthesis following the procedure of Jurczyk et al. [25]. Each oligonucleotide was purified by polyacrylamide gel electrophoresis (PAGE).

Annealing, extending and ligating the preselected DNA oligonucleotide fragments

The lyophilized synthetic fragments were resuspended in buffer (10 mM Tris-HCl, pH 8.0, 0.1 mM EDTA) to give stock solutions, each holding $10 \mu \mathrm{M}$ of DNA (by UV spectroscopy at $260 \mathrm{~nm}$ prior to dilution). For the annealing step, aliquots $(1 \mu \mathrm{L}$, containing each 10 pmol, ca. 125-250 ng of DNA) were combined from each of the stock solutions with $5 \times$ ISO buffer (8 $\mu \mathrm{L}, 25 \%$ PEG-6000, $500 \mathrm{mM}$ Tris-HCl, pH 7.5, $50 \mathrm{mM}$ $\mathrm{MgCl}_{2}, 50 \mathrm{mM}$ DTT, $\left.5 \mathrm{mM} \mathrm{NAD}{ }^{+}\right)$and diluted with water (40 $\mu \mathrm{L}$ final volume; final concentrations: $250 \mathrm{nM}$ each

Table 1: Oligonucleotides containing S (2'-deoxy-5-methylisocytidine) and B (2'-deoxyisoguanosine) used in this work.

\begin{tabular}{|c|c|}
\hline Name & Sequence (5'-3') \\
\hline 01KanR 1_66 & CACCATGAGCCATATTCAACGGGAAACGTCGAGGCCGCGATTAAATTCCAACATGGASGCSGASTT \\
\hline 02KanR 55_106 & SGASTGCCCGACBTTATCGCGAGCCCATTTATACCCATATAABTCBGCBTCC \\
\hline 03KanR 94_151 & SGTCGGGCABTCBGGTGCGACAATCTATCGCTTGTATGGGAAGCCCGASGCGCCBGAG \\
\hline 04KanR 141_193 & BACBTCBTTGGCBACGCTACCTTTGCCATGTTTCAGAAACAACTCSGGCGCBT \\
\hline 05KanR 181_242 & SGCCAASGASGTSACAGATGAGATGGTCAGACTAAACTGGCTGACGGABTTTATGCCSCTSC \\
\hline 06KanR 228_276 & TCBTCBGGBGTBCGGATAAAATGCTTGATGGTCGGBAGBGGCATAAAST \\
\hline 07KanR 265_318 & SACSCCSGASGATGCATGGTTACTCACCACTGCGATCCCCGGBAAAACBGCBTT \\
\hline 08KanR 306_362 & CBACAATBTTSTCBCCTGAATCAGGATATTCTTCTAATACCTGGAASGCSGTTTTSC \\
\hline 09KanR 349_407 & SGABAASATTGTSGATGCGCTGGCAGTGTTCCTGCGCCGGTTGCASTCGATTCCTGTST \\
\hline 10KanR 393_449 & GBGCGAGBCGAAATACGCGATCGCTGTTAAAAGGACAATTACABACAGGAATCGABT \\
\hline 11KanR 439_485 & TCGSCTCGCSCAGGCGCAATCACGAATGAATAACGGSTTGGTSGASG \\
\hline 12KanR 474_530 & CSTGSTCBACBGGCCAGCCATTACGCTCGTCATCAAAATCACTCGCBTCBACCAABC \\
\hline 13KanR 520_560 & SGTSGABCABGTCTGGAAAGAAATGCASAABCTSTTGCCBT \\
\hline 14KanR 546_604 & BTCBAGSGAGAABTCACCATGAGTGACGACTGAATCCGGTGAGAASGGCAABAGSTTBT \\
\hline 15KanR 592_657 & ASTTCTCBCTSGASAACCTTATTTTTGACGAGGGGAAATTAATAGGTTGTATTGASGTTGGACGBGT \\
\hline 16KanR 643_686 & CBAGBTCCTGGTASCGGTCTGCGATTCCGACSCGTCCAACBTCA \\
\hline 17KanR 673_734 & BTACCAGGASCTSGCCATCCTATGGAACTGCCTCGGTGAGTTTTCTCCSTCBTTACAGAABC \\
\hline 18KanR 720_778 & STTBTTCATBTCBGGATTATCAATACCATATTTTTGAAAAAGCCGSTTCTGTAASGABG \\
\hline 19KanR 765_832 & CSGASATGAASAABTTGCAGTTTCATTTGATGCTCGATGAGTTTTTCTAACAGGATCCGCBCGBCSAG \\
\hline 20KanR 822_863 & CTAGSGGSCGBTCSGTCCGTCCTGTCAGCTGCTBGSCGSGCG \\
\hline
\end{tabular}


oligonucleotide, 5\% PEG-6000, 100 mM Tris-HCl, pH 7.5, 10 $\mathrm{mM} \mathrm{MgCl} 2,10 \mathrm{mM}$ DTT, $1 \mathrm{mM} \mathrm{NAD}{ }^{+}$).

This mixture was then heated at $95{ }^{\circ} \mathrm{C}$ for $5 \mathrm{~min}$, and then slowly cooled $\left(0.1{ }^{\circ} \mathrm{C} /\right.$ second $)$ to $42{ }^{\circ} \mathrm{C}$. Aliquots $(5 \mu \mathrm{L})$ of the annealed mixture were transferred to a new tube, and then diluted with a mixture of enzymes (total volume of mixture was $15 \mu \mathrm{L}$ ) containing Phusion ${ }^{\mathrm{TM}}$ DNA polymerase (1 U, New England Biolabs, final $0.07 \mathrm{U} / \mu \mathrm{L}$ ), Taq DNA ligase (400 U, New England Biolabs, final $27 \mathrm{U} / \mu \mathrm{L}$ ), ISO buffer, and dNTPs (final $0.2 \mathrm{mM}$ ). The mixtures were then incubated at $48{ }^{\circ} \mathrm{C}$ for $60 \mathrm{~min}$, cooled and stored at $4{ }^{\circ} \mathrm{C}$.

The annealed-extended-ligated products were resolved on a $1 \%$ agarose gel (Figure 5). A major product appeared with a size of $\sim 863$ base pairs, the length of the fully assembled target L-DNA product.

\section{PCR amplification with conversion of the AEGIS- containing L-DNA product}

"Conversion PCR" [25,26] of the assembled product was then carried out using Taq Full DNA polymerase (Clontech), which lacks proofreading ability. This leads to the replacement of $\mathbf{S}: \mathbf{B}$ pairs by T:A pairs, presumably via the "minor enol tautomer" mechanism shown in Figure 3.

Two conditions were tested for the conversion PCR. The first included a small amount of 2'-d-isoGTP (dBTP, ChemGenes, $0.3 \mu \mathrm{L}$ of a $10 \mathrm{mM}$ stock, $3 \mathrm{nmol}$ per assay, final concentration $60 \mu \mathrm{M})$ to complement the 2'-d-isoC in the template. In the presence of $\mathrm{dBTP}$, the conversion requires two cycles. In the first, dBTP is incorporated opposite template dS, while dTTP is mismatched opposite template $\mathrm{d} \mathbf{B}$, presumably via its minor tautomer. In the second cycle, conversion is completed by mismatching $\mathrm{dT}$ opposite the minor enol $\mathrm{dB}$ in the newly synthesized template (Figure 3). Thus, the use of dBTP allows the PCR to proceed without requiring the polymerase to mismatch a standard nucleotide opposite template $\mathrm{d} \mathbf{S}$, a mismatch that is not assisted by any minor tautomer.

A second incubation omitted $\mathrm{dBTP}$. This required that in the first cycle, the polymerase must mismatch both dT opposite $\mathrm{dB}$ and $\mathrm{dA}$ opposite $\mathrm{d} \mathbf{S}$. As shown, these conditions produced lower yields of product, and are not recommended. Nevertheless, this result is consistent with our model for the conversion of $\mathbf{S}: \mathrm{B}$ pairs to T:A pairs.

The annealed-extended-ligated (AEL) sample was used directly in the conversion PCR, without clean-up or dilution. Aliquots of the AEL sample $(2 \mu \mathrm{L})$ were added to the pre-mix $(48 \mu \mathrm{L})$ of reagents and enzymes (Supporting Information File 1, Table
S2) optionally containing dBTP in PCR tubes $(200 \mu \mathrm{L})$. Water was added for the negative controls. The PCR cycling program was: $95^{\circ} \mathrm{C}$ for $2 \mathrm{~min}$, followed by 30 cycles of $95^{\circ} \mathrm{C}$ for $40 \mathrm{~s}$, $55^{\circ} \mathrm{C}$ for $20 \mathrm{~s}$, and $72{ }^{\circ} \mathrm{C}$ for $2 \mathrm{~min}$, with a final extension at $72{ }^{\circ} \mathrm{C}$ for $10 \mathrm{~min}$.

As the reverse primer was designed to be upstream of the terminal AEGIS bases, the final PCR product was $14 \mathrm{bp}$ shorter than the initial construct. The bands corresponding to the 849 bp amplicons were excised and purified with the Wizard SV Gel Clean-up System (Promega). Concentrations of amplicon DNA were estimated by their absorbance at $260 \mathrm{~nm}$.

\section{Secondary PCR}

The L-DNA construct was then prepared for insertion into plasmid vector. Because Taq DNA polymerase adds untemplated 3'-adenosines that interfere with incorporation of insert into a directional expression vector, a second PCR was carried out with a non-Taq polymerase (PrimeSTAR ${ }^{\mathrm{TM}}$, Clontech) using the gel-purified amplicon from the first PCR as its template. Three samples were prepared for secondary PCR: the product prepared by conversion PCR with $\mathrm{dBTP}$, the product prepared by conversion PCR without $\mathrm{dBTP}$, and a negative control lacking template. PCR reagents were pre-mixed (Supporting Information File 1, Table S3). Aliquots $(49 \mu \mathrm{L})$ were delivered to $0.2 \mathrm{~mL}$ PCR tubes, template or water $(1 \mu \mathrm{L})$ was added to give a final volume of $50 \mu \mathrm{L}$, and PCR was initiated following the program recommended by the supplier of the PrimeSTAR polymerase: hot start at $95{ }^{\circ} \mathrm{C}$ for five min followed by 30 cycles of: $98{ }^{\circ} \mathrm{C}$ for $10 \mathrm{~s}$, then $68{ }^{\circ} \mathrm{C}$ for $20 \mathrm{~s}$. The amplicon was recovered using the QIAquick PCR Purification kit (Qiagen).

\section{Incorporation into the vector}

Each cleaned-up amplicon $(+\mathrm{d} \mathbf{B}$ and $-\mathrm{d} \mathbf{B}, 4 \mu \mathrm{L})$ was mixed with TOPO pET101 directional expression vector $(1 \mu \mathrm{L}$, Invitrogen) and salt solution ( $1 \mu \mathrm{L}$, provided with vector) according to the manufacturer's recommendation, incubated at room temperature for $10 \mathrm{~min}$, then transferred to wet ice until used to transform competent cells. The insert/vector mixtures were used to transform BL21 Star (DE3, Invitrogen) cells using heatshock following the manufacturer's instructions. For each sample type, a fraction of the DNA/vector $(3 \mu \mathrm{L})$ was added to cells (50 $\mu \mathrm{L}$, provided with vector kit), and the mixture was incubated on ice for $30 \mathrm{~min}$. The cells were then shocked by heating $\left(42{ }^{\circ} \mathrm{C}, 35 \mathrm{~s}\right)$ in a water bath. Media (SOC from Invitrogen, $250 \mu \mathrm{L}$, provided with vector kit) was then added, and the mixture was placed on ice for $5 \mathrm{~min}$. The mixtures were then transferred to Falcon tubes $(15 \mathrm{~mL})$ and incubated $\left(37^{\circ} \mathrm{C}\right.$, shaking) for $1 \mathrm{~h}$. The entire volumes were then transferred by pipetting to pre-warmed $\left(37^{\circ} \mathrm{C}\right) \mathrm{LB}$ medium containing ampi- 
cillin $(100 \mu \mathrm{g} / \mathrm{mL}$, without kanamycin) and incubated overnight $\left(37^{\circ} \mathrm{C}\right)$. These conditions allowed cells containing the plasmid to grow, whether or not the plasmid contained a functioning inserted gene encoding kanamycin resistance (or, for that matter, any insert at all). To assess whether the assembly had produced a gene able to confer kanamycin resistance, these cells were subcultured in shaken growth medium containing kanamycin and isopropyl $\beta$-D-1-thiogalactopyranoside (IPTG). Continued growth was observed, and viability was measured by following an increase in optical density (OD) as a function of time. Negative controls (cells that lacked the kanamycin resistance gene) did not survive overnight incubation in the presence of kanamycin.

Additionally, each insert/vector mixture $(+\mathrm{d} \mathbf{B}$ and $-\mathrm{dB}, 2 \mu \mathrm{L}$ each) was used to transform TOP10 competent cells $(25 \mu \mathrm{L}$ each, provided with vector kit), a more stable cell line for culturing and maintenance. The cells/DNA mixture was incubated on wet ice for $8 \mathrm{~min}$, shocked at $42{ }^{\circ} \mathrm{C}$ for $35 \mathrm{~s}$, and returned to the ice briefly. Room temperature SOC media $(250 \mu \mathrm{L})$ was immediately added and the cells transferred to a culture tube and incubated at $37^{\circ} \mathrm{C}$ with shaking for $1 \mathrm{~h}$. After incubation the TOP10 cells were divided between two LB/ Ampicillin $(100 \mu \mathrm{g} / \mathrm{mL})$ agar plates $(100$ and $175 \mu \mathrm{L})$ and incubated at $37{ }^{\circ} \mathrm{C}$ overnight. After incubation the plates were stored at $4{ }^{\circ} \mathrm{C}$ until prepared for sequencing reactions.

\section{Quantitative estimates of the level of success of the OligArch L-DNA construction}

Cells from the growth in shaken culture in the presence of kanamycin were used to inoculate a new culture $(3 \mathrm{~mL})$ of $\mathrm{LB}$ medium containing ampicillin $(100 \mu \mathrm{g} / \mathrm{mL})$ and incubated with shaking at $37{ }^{\circ} \mathrm{C}$ until OD reached 1.4 . Volumes $(25$ and $50 \mu \mathrm{L})$ of this culture were then plated (with appropriate serial dilutions) on agar containing ampicillin alone (to count cells that had been transformed regardless of the success of the OligArch assembly), kanamycin and isopropyl $\beta$-D-thiogalactoside (IPTG, $1 \mathrm{mM}$, which induced the expression of any kanamycin resistance gene carried by the plasmid), and kanamycin without IPTG (the negative control). Without IPTG, any kanamycin resistance genes are not induced, and no cells were expected to survive kanamycin.

\section{Sequencing the L-DNA construct that confers kanamycin resistance}

To prepare plasmids for sequencing of cells with selective pressure to contain the insert that correctly encodes for kanamycin resistance, cells previously found to be viable in shaken culture in the presence of kanamycin were plated on LB/agar plates containing kanamycin $(100 \mu \mathrm{g} / \mathrm{mL})$ and IPTG $(1 \mathrm{mM})$. Single colonies were then used to inoculate cultures grown in the pres- ence of ampicillin alone. Plasmids were harvested with the Zyppy ${ }^{\text {TM }}$ Miniprep kit (Zymo Research) and were sent out to be sequenced with vector primers (T7 Terminator and T7 Promoter long). The sequences obtained were then analyzed (Supporting Information File 1, Table S4).

To prepare plasmids with no selective pressure, which would potentially include inserts that contained assembly errors or base substitutions arising during PCR conversion other than S:T and $\mathbf{B}: \mathrm{C}$, colonies from the TOP10 culture plates were used to inoculate cultures grown in the presence of ampicillin alone. Plasmids were harvested as described above and sequenced with vector primers (BioBasic).

\section{Supporting Information}

\section{Supporting Information File 1}

Additional Information.

[http://www.beilstein-journals.org/bjoc/content/

supplementary/1860-5397-10-245-S1.pdf]

\section{Acknowledgements}

For support of this work, we are indebted to the DARPA Foundries program (HR0011-12-C-0064) and, via Firebird Biomolecular Sciences LLC, the Office of the Secretary of Defense under its program entitled "Design Automation Software for DNA-based Nano-Sensor Architecture" (W911NF-12C-0059). The basic research that made this work possible was funded by the Defense Threat Reduction Agency under its basic research program, including grant HDTRA1-13-1-0004.

\section{References}

1. Letsinger, R. L.; Mahadevan, V. J. Am. Chem. Soc. 1965, 87, 3526-3527. doi:10.1021/ja01093a058

2. Letsinger, R. L.; Mahadevan, V. J. Am. Chem. Soc. 1966, 88, 5319-5324. doi:10.1021/ja00974a053

3. Beaucage, S. L.; Caruthers, M. H. Tetrahedron Lett. 1981, 22, 1859-1862. doi:10.1016/S0040-4039(01)90461-7

4. Pennisi, E. Science 2011, 333, 147. doi:10.1126/science.333.6039.147

5. Caruthers, M. H.; Kleppe, K.; van de Sande, J. H.; Sgaramella, V.; Agarwal, K. L.; Büchi, H.; Gupta, N. K.; Kumar, A.; Ohtsuka, E.; RajBhandary, U. L.; Terao, T.; Weber, H.; Yamada, T.; Khorana, H. G. J. Mol. Biol. 1972, 72, 475-492. doi:10.1016/0022-2836(72)90158-1

6. Anand, N.; Bindra, J. S.; Ranganathan, S. Art in Organic Synthesis; Wiley-Interscience: New York, 1970; p 198.

7. Gibson, D. G.; Young, L.; Chuang, R.-Y.; Venter, J. C.; Hutchison, C. A., III; Smith, H. O. Nat. Methods 2009, 6, 343-345. doi:10.1038/nmeth.1318

8. Edge, M. D.; Greene, A. R.; Heathcliffe, G. R.; Meacock, P. A.; Schuch, W.; Scanlon, D. B.; Atkinson, T. C.; Newton, C. R.; Markham, A. F. Nature 1981, 292, 756-762. doi:10.1038/292756a0 
9. Nambiar, K. P.; Stackhouse, J.; Stauffer, D. M.; Kennedy, W. P.; Eldredge, J. K.; Benner, S. A. Science 1984, 223, 1299-1301. doi:10.1126/science. 6322300

10. Presnell, S. R.; Benner, S. A. Nucleic Acids Res. 1988, 16, 1693-1702. doi:10.1093/nar/16.5.1693

11. Gibson, D. G.; Benders, G. A.; Andrews-Pfannkoch, C.;

Denisova, E. A.; Baden-Tillson, H.; Zaveri, J.; Stockwell, T. B.; Brownley, A.; Thomas, D. W.; Algire, M. A.; Merryman, C.; Young, L.; Noskow, V. N.; Glass, J. I.; Venter, J. C.; Hutchison, C. A., III; Smith, H. O. Science 2008, 319, 1215-1220. doi:10.1126/science.1151721

12. Annaluru, N.; Muller, H.; Mitchell, L. A.; Ramalingam, S.; Stracquadanio, G.; Richardson, S. M.; Dymond, J. S.; Kuang, Z.; Scheifele, L. Z.; Cooper, E. M.; Cai, Y.; Zeller, K.; Agmon, N.; Han, J. S.; Hadjithomas, M.; Tullman, J.; Caravelli, K.; Cirelli, K.; Guo, Z.; London, V.; Yeluru, A.; Murugan, S.; Kandavelou, K.; Agier, N.; Fischer, G.; Yang, K.; Martin, J. A.; Bilgel, M.; Bohutskyi, P.; Boulier, K. M.; Capaldo, B. J.; Chang, J.; Charoen, K.; Choi, W. J.; Deng, P.; DiCarlo, J. E.; Doong, J.; Dunn, J.; Feinberg, J. I.; Fernandez, C.; Floria, C. E.; Gladowski, D.; Hadidi, P.; Ishizuka, I.; Jabbvari, J.; Lau, C. Y. L.; Lee, P. A.; Li, S.; Lin, D.; Linder, M. E.; Ling, J.; Liu, J.; Liu, J.; London, M.; Ma, H.; Mao, J.; McDade, J. E.; McMillan, A.; Moore, A. M.; Oh, W. C.; Ouyang, Y.; Patel, R.; Paul, M.; Paulsen, L. C.; Quu, J.; Rhee, A.; Rubashkin, M. G.; Soh, I. Y.; Sotuyo, N. E.; Srinivas, V.; Suarez, A.; Wong, A.; Wong, R.; Yie, W. R.; Xu, Y.; Yu, A. T.; Koszul, R.; Bader, J. S.; Boeke, J. D.; Chandrasegaran, S. Science 2014, 344, 55-58. doi:10.1126/science. 1249252

13. Leduc, S. In La Biologie Synthétique; Poinat, A., Ed.; Etudes de Biophysique: Paris, 1912.

14. Rawis, R. L. Chem. Eng. News 2000, 78 (17), 49-53. doi:10.1021/cen-v078n017.p049

15. Ball, P. Nature 2004, 431, 624-626. doi:10.1038/431624a

16. Gibbs, W. W. Sci. Am. 2004, 290, 74-81.

17. Benner, S. A.; Sismour, A. M. Nat. Rev. Genet. 2005, 6, 533-543. doi:10.1038/nrg1637

18. Benner, S. A. Acc. Chem. Res. 2004, 37, 784-797. doi:10.1021/ar040004z

19. Benner, S. A.; Yang, Z.; Chen, F. C. R. Chim. 2011, 14, 372-387. doi:10.1016/j.crci.2010.06.013

20. Yang, Z.; Chen, F.; Alvarado, J. B.; Benner, S. A. J. Am. Chem. Soc. 2011, 133, 15105-15112. doi:10.1021/ja204910n

21. Bradley, K. M.; Benner, S. A. Beilstein J. Org. Chem. 2014, 10, 1826-1833. doi:10.3762/bjoc.10.192

22. Gibson, D. G. Methods Enzymol. 2011, 498, 349-361. doi:10.1016/B978-0-12-385120-8.00015-2

23. Gibson, D. G. Nucleic Acids Res. 2009, 37, 6984-6990. doi:10.1093/nar/gkp687

24. SantaLucia, J., Jr. Proc. Natl. Acad. Sci. U. S. A. 1998, 95, 1460-1465. doi:10.1073/pnas.95.4.1460

25. Jurczyk, S. C.; Kodra, J. T.; Rozzell, J. D., Jr.; Benner, S. A.; Battersby, T. R. Helv. Chim. Acta 1998, 81, 793-811. doi:10.1002/hlca.19980810502

26. Yang, Z.; Durante, M.; Glushakova, L. G.; Sharma, N.; Leal, N. A.; Bradley, K. M.; Chen, F.; Benner, S. A. Anal. Chem. 2013, 85 , 4705-4712. doi:10.1021/ac400422r

\section{License and Terms}

This is an Open Access article under the terms of the Creative Commons Attribution License

(http://creativecommons.org/licenses/by/2.0), which permits unrestricted use, distribution, and reproduction in any medium, provided the original work is properly cited.

The license is subject to the Beilstein Journal of Organic Chemistry terms and conditions:

(http://www.beilstein-journals.org/bjoc)

The definitive version of this article is the electronic one which can be found at:

doi:10.3762/bjoc. 10.245 